\title{
MONUMENTO HISTÓRICO Y PAISAJE. ESTUDIO Y PROPUESTA DE ORDENACIÓN DE LA TRAZA IGNORADA DEL ACUEDUCTO DE SEGOVIA*
}

\author{
Rafael Mata Olmo \\ Daniel Ferrer Jiménez \\ Departamento de Geografía. Universidad Autónoma de Madrid \\ rafael.mata@uam.es
}

\section{RESUMEN}

El texto aborda el estudio de las relaciones entre monumento histórico, territorio y paisaje, tomando como ejemplo el acueducto de Segovia en su conjunto, desde su nacimiento en la sierra de Guadarrama hasta la ciudad de Segovia. El artículo se enmarca en los nuevos planteamientos sobre patrimonio territorial y paisaje. Estudia y valora los paisajes que el acueducto atraviesa, especialmente los del tramo no monumental. El texto analiza después el planeamiento urbanístico y territorial en relación con el monumento y diagnostica el estado de abandono y deterioro del acueducto en el área periurbana de Segovia. El artículo termina con la formulación de una propuesta abierta para la conservación integral del Acueducto de Segovia y el disfrute de sus paisajes.

Palabras clave: Acueducto de Segovia, patrimonio, paisaje, Planificación territorial, uso público.

\section{ABSTRACT}

The text studies the relationships between the historical monument, its territory and landscape, taking as an example the Aqueduct of Segovia as a whole, from its source in the Sierra of Guadarrama to the city of Segovia. The framework of this paper is the new planning of the regional and landscape heritage. It examines and reviews the different landscapes

Fecha de recepción: mayo 2011.

Fecha de aceptación: diciembre 2012.

* Investigación desarrollada dentro del proyecto CSO2012-39564-C07-03, financiado por la Dirección General de Investigación Científica y Técnica, Ministerio de Economía y Competitividad del Gobierno de España. 
along the aqueduct, especially the non-monumental sector. In addition, this paper analyses the regional and urban planning in relation to the monument and diagnoses the state of abandonment and deterioration of the Aqueduct in the peri-urban area of Segovia. Finally, an open proposal is put forward in order to achieve the entire conservation of the Aqueduct of Segovia and to enjoy its landscapes.

Key words: Aqueduct of Segovia, heritage, landscape, territorial planning, public use.

\section{MONUMENTO, TERRITORIO Y PAISAJE. A PROPÓSITO DEL ACUEDUCTO DE SEGOVIA}

Existe un reconocimiento unánime de que el Acueducto de Segovia constituye una obra singular y única de la ingeniería romana, la más importante de España para algunos expertos (Jurado Jiménez, 2001) y de las más notables del Imperio. Como «el más airoso de todos los que construyeron los romanos», lo califica Carlos Fernández Casado en su fundamental tratado sobre los acueductos romanos en España, de 1972, «duradero como muy pocas construcciones, que podría seguir cumpliendo su función primaria de llevar agua rodada y libre» (Fernández Casado, 1972; ed. 2008: 53).

A la importancia histórica y prolongada funcionalidad hidráulica de este «peine del tiempo», en palabras de Ramón Gómez de la Serna, se suma el valor estético y monumental de su fábrica y la armónica integración en la forma e imagen urbana de una ciudad medieval, notable también, como Segovia. «El ingente acueducto, (...) como formidable elemento morfológico que ha condicionado siempre el urbanismo segoviano (...), dirige la atención, conduce la mirada por el viejo camino del agua, inevitablemente hacia la peña y retrotrae el pensamiento a la antigüedad romana» (Martínez de Pisón, 1976: 13-14).

Lógicamente la parte más apreciada y representada del acueducto, la que ha concitado más interés de estudio y conservación, y también la más «valorizada» por la que Fraçoise Choay ha denominado «ingeniería cultural» (Choay, 1992, ed. 2007: 194), es la traza aérea de la obra. Sin embargo, su carácter de infraestructura conductora de agua para abastecimiento de la ciudad requiere la consideración y el tratamiento patrimonial de la construcción en su integridad, desde las nacientes en el azud del río Frío, en el corazón de la umbría de la sierra de Guadarrama, hasta su terminación en el Alcázar de Segovia.

Esta consideración unitaria del acueducto fortalece, en primer lugar, el sentido de «monumento histórico», con la profundidad que a comienzos del siglo XX atribuyó a esta noción Alois Riegl, frente al concepto previo de simple «monumento». Como destaca la propia Choay, glosando al autor austriaco, el monumento es una creación deliberada (gewollte), conmemorativa (del latín monumentum, derivado a su vez de monere -advertir, recordar-), y está directamente relacionado con la memoria, cuya función ha sido asumida a priori. El monumento histórico, sin embargo, no ha sido querido (ungewollte) y creado como tal inicialmente; «(...) se constituye a posteriori, a través de las miradas convergentes del historiador y del aficionado» (Choay, 1993: 16), lo que le atribuye más fuerza interpretativa y, frecuentemente, mayor conexión con el contexto en el que fue originariamente construido.

Pero la consideración unitaria de una obra histórica de estas características supone también -o debería suponer- su inserción en el territorio y el diálogo con el paisaje que este 
camino del agua ha recorrido secularmente y que hoy, pese a su abandono, lo marca y cualifica. Esta proyección del elemento patrimonial al paisaje y la complicidad necesaria entre el acueducto y su entorno, que dignifica a ambos, constituye un buen ejemplo de la apertura semántica de la noción clásica de patrimonio y su expansión, en lo material, del monumento al conjunto histórico y del conjunto al territorio interpretado a través del paisaje (Mata Olmo, 2010: 45 y ss.). Tal proyección reclama políticas y acciones de protección, mejora y uso público para el acueducto en su unidad y para el territorio contextual que lo acoge.

Ciertamente la inserción en el territorio de la modesta conducción que durante casi dos milenios ha abastecido de agua a Segovia desde el Guadarrama, antes de alcanzar el tramo aéreo y monumental que salva la vaguada urbana del Azoguejo, no tiene la fuerza interpretativa -material y perceptiva- de otros monumentos y sitios históricos con respecto a sus entornos, como han puesto de manifiesto recientes trabajos en España sobre el paisaje de los conjuntos arqueológicos (Caballero Sánchez y Zoido Naranjo, 2008; Centro de Estudios Paisaje y Territorio, 2011) o de determinados sitios históricos y simbólicos, como la Sierra de los Molinos cervantinos en Campo de Criptana (Mata Olmo y Galiana Martín, 2008). No obstante, el territorio que la cacera recorre está marcado por su huella, y la huella sigue un viaje que une Segovia y su Sierra con el vínculo del agua domesticada. Además, pese a su reducida longitud, el canal atraviesa paisajes muy representativos del tránsito entre el Sistema Central y la meseta. Estas razones justifican sobradamente la atención que merece la traza ignorada del acueducto y el estudio llevado a cabo con ese motivo.

\section{UN ESTUDIO PARA LA INTEGRACIÓN DEL ACUEDUCTO EN SU ENTORNO TERRITORIAL A TRAVÉS DEL PAISAJE}

El sobresaliente interés y los valores históricos, arqueológicos, ingenieriles y estéticos del acueducto de Segovia, y su buen estado de conservación, motivaron la temprana declaración de su parte aérea como Monumento Histórico-Artístico en 1884 (Real Orden de 11 de octubre). En la actualidad es Bien de Interés Cultural, conforme a la legislación de patrimonio vigente, y junto a la ciudad vieja de Segovia está inscrito, desde el 6 de diciembre de 1985, en la Lista del Patrimonio Mundial de la UNESCO.

Su conservación ha sido objeto de preocupación, investigación y, en algunos casos, de intervenciones, no exentas de polémica, «como ocurre siempre que se interviene en una obra histórica de la importancia del Acueducto de Segovia» (Fernández Troyano, 2007: 9) ${ }^{1}$. Al paso del tiempo y a impactos naturales (acción de aves y plantas enraizadas en sus sillares, heladas y fuertes cambios térmicos) se suma ahora «un ambiente más contaminado, que ha provocado una importante aceleración del deterioro de los sillares, como directamente se desprende de todos los estudios realizados» (Jurado Jiménez, 2001: 17).

En 2006, el Acueducto de Segovia fue incluido en la Lista de Monumentos en Peligro de la World Monuments Fund (WMF) ${ }^{2}$, abriéndose un periodo de reflexión sobre la situación

1 Se refiere Leonardo Fernández Troyano concretamente al «Proyecto de Reparación y Consolidación del Acueducto de Segovia», terminado en 1967 y que llevó a cabo su padre, Carlos Fernández Casado, de lo que en buena medida es fruto su obra fundamental ya citada, Acueductos romanos en España (1972; segunda edición, 2008).

2 La WMF es una entidad privada norteamericana, sin ánimo de lucro, que dirige sus esfuerzos desde 1965 hacia la conservación y restauración de obras de arte y monumentos singulares en todo el mundo. http://www.wmf.org. 
real de la construcción. Resultan de interés las razones que llevaron a WMF a la inclusión del acueducto en la citada lista, pues como se puede comprobar, hay motivos directamente ligados a la fábrica del monumento, pero también a su entorno y a la integridad de la traza. Estos son los principales problemas y amenazas que motivaron su designación:

- Falta de mantenimiento, pérdidas de agua y elevada contaminación.

- Reducción de la estabilidad de la estructura, comprometida por el deterioro del granito.

- Daños provocados por el anidamiento de aves.

- Falta de protección del trazado en su integridad.

- Presión urbanística que amenaza el entorno y paisajes del acueducto.

- Pérdida irreversible de parte del trazado por la construcción del AVE.

- Impacto negativo de determinadas prácticas urbanas (aparcamiento, conciertos, actos multitudinarios...).

- Falta de colaboración entre las distintas administraciones.

Con objeto de corregir los problemas señalados, de prevenir el deterioro del monumento en su conjunto y de avanzar hacia un Plan Director para la Conservación del Acueducto de Segovia, que defina una estrategia global y asegure su mantenimiento y seguimiento, se iniciaron en 2009 distintos trabajos de ingeniería, geología y arqueología, a cargo de reconocidos especialistas.

En ese contexto, WMF consideró pertinente un denominado «Estudio de integración territorial y paisajística del trazado del acueducto de Segovia», encargado a la Universidad Autónoma de Madrid y en el que participaron los autores de este texto. No se trata de un estudio arqueológico o técnico del elemento monumental, aunque la investigación realizada ha permitido conocer su estado de conservación. El objetivo fue desde el principio caracterizar y valorar su integración en el territorio desde las nacientes serranas a la ciudad de Segovia, con la perspectiva que preconiza el Convenio Europeo del Paisaje (Consejo de Europa, 2000), particularmente en el recorrido rural y periurbano del canal, a lo largo del cual la ignorancia y la desidia han provocado ya -y la situación puede agravarse hasta llegar a ser irreversible- abandono, deterioro y hasta destrucción parcial.

La originalidad del estudio radica, pues, en el tratamiento del acueducto completo, como elemento lineal y continuo de algo más de $16 \mathrm{~km}$, en su relación material y visual con el territorio por el que discurre, desde su origen en el azud del río Frío, a $1.255 \mathrm{~m}$ de altitud, en los pinares de la Acebeda, hasta la peña del Alcázar de Segovia, a 985 m. Estos son las características más significativas de su trazado (Marqués Martín, 2010):

- Longitud total: $16.220 \mathrm{~m}$. (desde su azud, incluida la presa de 1929, hasta la puerta del Alcázar segoviano):

- Desde el azud (incluida la presa del 1929) al primer desarenador urbano: 13.393 $\mathrm{m}$.

- Tramo comprendido entre los dos desarenadores (ambos incluidos): $794 \mathrm{~m}$.

- A partir del desarenador de San Gabriel hasta la plaza de Avendaño (tramo monumental): $776 \mathrm{~m}$.

- Canal soterrado (desde la calle Obispo Gandasegui hasta la puerta del Alcázar): $1.220 \mathrm{~m}$. 
- División histórica del Acueducto de Segovia en tres tramos bien diferenciados:

1. Zona extraurbana, donde se sitúa la captación y parte de la conducción.

2. Tramo periurbano. Conducción.

3. Trazado urbano. Conducción y distribución.

3.1. Acueducto aéreo y monumental.

\subsection{Canal soterrado.}

Con estas líneas sintetiza Carlos Fernández Casado el curso no aéreo del acueducto en su valiosa «descripción» de la obra:

«La conducción de aguas para el abastecimiento de Segovia no es de las más importantes de entre las que construyeron los romanos, pues tiene una longitud de unos $18 \mathrm{~km}$ y su caja es de 30 por $30 \mathrm{~cm}$ (1 por 1 pie, aproximadamente). Tomaba el agua en el nacimiento del río Frío, cerca de la fuente de la Fuenfría, que da nombre al río y al puerto de paso de la Sierra por ese lugar, derivándola mediante presa romana, que fue reconstruida cuando los Reyes Católicos. Desciende en canal abierto contorneando laderas rocosas de unos $9 \mathrm{~km}$ para llegar al pinar de Valsaín, cruzando la carretera que fue camino real de San Indefonso junto a la antigua venta de Santillana, y ya desde allí enterrada por el llano, y advertida por una serie de registros, cuya fábrica parece del siglo XV, llega hasta una construcción elevada denominada el Caserón, que debió ser castellum aquae de la primitiva conducción. A partir de esta construcción iba la construcción sobre muro hasta la segunda caseta o desarenador que describimos más adelante» (Fernández Casado, 1972, 2. ${ }^{\text {a }}$ ed. 2008: 56).

Sobre el trazado aéreo y monumental, el más valioso y apreciado, el estudio llevado a cabo ha consistido, como se expondrá más adelante, en el análisis visual del acueducto dentro del tejido urbano de Segovia para definir y evaluar el estado actual de las condiciones visuales y los impactos que afectan al monumento y a sus alrededores, con objeto de mejorar la calidad de la escena, el paisaje de la ciudad en el ámbito del acueducto y, como resultado, realzar el interés arqueológico, histórico y arquitectónico de las vistas, fomentando un mayor y mejor reconocimiento del significado patrimonial de las mismas (English Heritage, 2011) .

En cuanto a la «zona extraurbana» y al «tramo periurbano», el estudio se ha centrado en los siguientes asuntos:

- Caracterización y estado de conservación del área que surca el canal, destacando la presencia de otros elementos construidos o naturales de interés patrimonial contiguos o próximos, e incorporando la perspectiva paisajística, de acuerdo con la concepción de paisaje del Convenio de Florencia.

- Tratamiento del acueducto en la planificación territorial a dos escalas: el Plan General de Ordenación Urbana (PGOU) de Segovia y las Directrices de Ordenación del Territorio de Segovia y su Entorno (DOTSE).

- Estimación del potencial del trazado del acueducto para articular un programa de uso público y de interpretación patrimonial y paisajística, y formulación de propuestas de protección, gestión y mejora, de acuerdo también con los planteamientos del citado Convenio del Paisaje. 


\section{LOS PAISAJES DEL ACUEDUCTO: PERCEPCIÓN DE NATURALEZA E HISTORIA EN LA TRANCISIÓN DE LA SIERRA A LA MESETA}

Siguiendo el curso kilométrico del acueducto entre el azud y la ciudad de Segovia es posible recorrer, en apenas $14 \mathrm{~km}$, un amplio y diverso repertorio de paisajes castellanos,

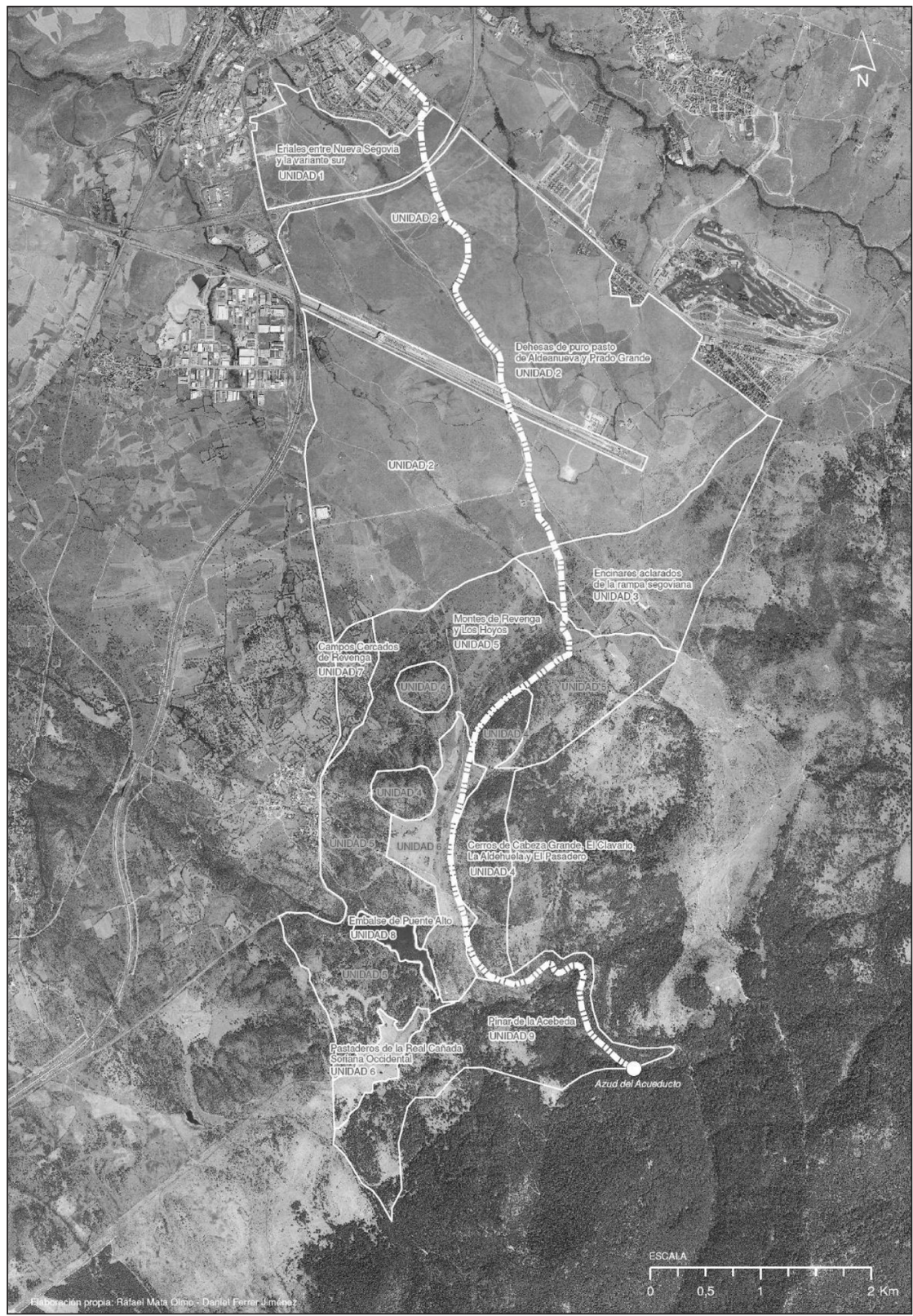


representativos de la vertiente segoviana de la sierra de Guadarrama y de su rampa. Resultado del trabajo realizado se han identificado y caracterizado nueve paisajes, con morfologías contrastadas, cuyo carácter responde a particulares relaciones entre elementos de la naturaleza (fundamentalmente, topografía, altitud, clima local y cubierta vegetal natural) y modos de apropiación y de aprovechamiento humano de los recursos, de claras raíces históricas. A ello se suman, gracias a la pendiente descendente, muy buenas condiciones de visibilidad y observación desde el propio canal o desde puntos próximos ubicados sobre pequeños resaltes del terreno.

Comenzando en el azud del río Frío y descendiendo hasta las puertas del área urbana de Segovia, se han distinguido las siguientes unidades de paisaje: «Pinares de la Acebeda»; «Entorno del embalse de Puente Alto»; «Dehesas de fresnos y campos cercados de Revenga»; «Pastaderos de la Real Cañada Soriana Occidental»; «Montes de Revenga y los Hoyos»; «Cerros de Cabeza Grande, El Calvario y La Aldehuela»; «Encinares aclarados de la rampa segoviana», «Dehesas de puro pasto de Andeanueva y Pradogrande» y «Eriales entre Nueva Segovia y la variante Sur». A continuación se recogen algunas de sus principales características geográfico-físicas, históricas y visuales.

\section{Pinares de la Acebeda}

Los pinares de la Acebeda forman parte del histórico conjunto forestal de los Montes de Valsaín, extendiéndose por los términos municipales de San Ildefonso, La Granja y Segovia. Todas las masas de Valsaín pertenecieron en sus orígenes medievales a la ciudad de Segovia,

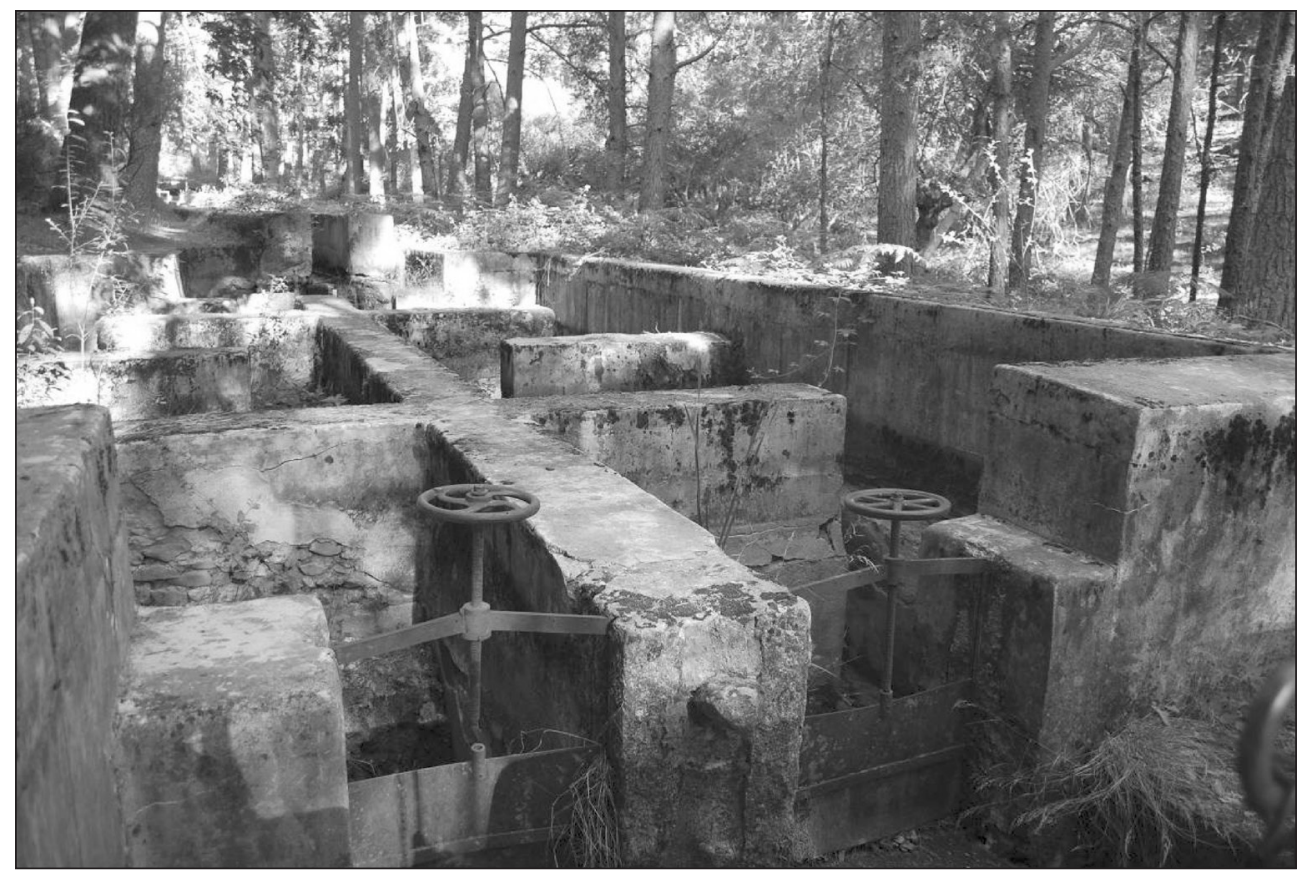

El azud del acueducto 
a la Junta de Nobles Linajes y al Común de la Ciudad y Tierra, hasta ser adquiridos mediante compra, en el año 1761, por la Corona, agrupando también las «matas» Pirón y Riofrío (Manuel Valdés, 2008:3). Desde estas fechas, y durante un largo periodo de tiempo, la propiedad estuvo vinculada a la Corona ${ }^{3}$. Durante la II Republica formaron parte del Patrimonio del Estado, antes de pasar, tras la Guerra Civil, al Patrimonio Nacional. En la actualidad su titularidad corresponde al Organismo Autónomo Parques Nacionales.

El elevado valor intrínseco de este espacio desde el punto de vista geomorfológico, ecológico y paisajístico, que le ha hecho merecedor de un alto grado de protección ${ }^{4}$, se acrecienta en la medida en que esta unidad de paisaje tan característica acoge el propio azud del acueducto y los primeros kilómetros de su conducción.

En cuanto al uso público, el tramo que discurre por la Acebeda, si bien es cierto que no permite grandes panorámicas por la propia naturaleza boscosa del paisaje, brinda sin embargo la oportunidad de recorrer y reconocer un bosque maduro de pino silvestre (Pinus sylvestris), históricamente connotado, y en el que tienen cabida también otras formaciones arboladas, como rebollares y encinares. El fácil acceso al azud desde el embalse de Revenga potencia las cualidades interpretativas y recreativas del área.

\section{Entorno del embalse de Puente Alto}

Se ha considerado que esta pequeña lámina de agua, próxima a la Cañada Real Soriana Occidental, a la vereda de El Sobornal y al propio trazado del acueducto, constituye una unidad de paisaje con entidad propia, así como un elemento de considerable importancia ambiental, pese a tratarse de una masa de agua creada artificialmente.

La pequeña presa de gravedad embalsa las aguas del arroyo del río Frío o de la Acebeda, abasteciendo la actual conducción de agua potable de Segovia y Hontoria, así como de otras localidades al oeste, como Navas de Riofrío, La Losa, Ortigosa del Monte, Otero de Herreros, Los Ángeles de San Rafael o El Espinar. También Revenga, pueblo próximo, se abastece del embalse de Puente Alta, complementando el suministro derivado desde la cacera de la Acebeda o cacera del Acueducto.

Destaca en el paisaje la profusa vegetación que coloniza las riberas del embalse, con presencia de pinos (Pinus sylvestris), encinas (Quercus ilex) y algunos robles (Quercus pyrenaica), así como la diversidad cromática y la capacidad especular que introduce la lámina de agua.

En la zona de alimentación del mismo, se aprecia una interesante vegetación de ribera, compuesta por sauces (Salix sp.), servales (Sorbus aucuparia), fresnos (Fraxinus angustifolia) y otras especies hidrófilas. La unidad presenta una gran importancia para las aves, quedando englobada en la ZEPA Sierra de Guadarrama.

3 En rigor, con algunas excepciones, ya que existieron adquisiciones de particulares tras la promulgación de la ley de desvinculación y venta de bienes de la Corona y con anterioridad a la Restauración, momento en el que, salvo las matas de Pirón, La Sauca y Navalosar, todos los montes son recuperados (Manuel Valdés, 2008: 8).

4 La singularidad y los valores ambientales del Pinar de la Acebeda justifican que en 1987 éste fuera incluido en la Red Natura 2000 mediante la doble consideración de Lugar de Importancia Comunitaria (LIC) y Zona de Especial Protección para las Aves (ZEPA). 


\section{Dehesas de fresnos y campos cercados de Revenga}

Al norte de la localidad de Revenga, sobre suelos hidromorfos, con una gran capacidad de retención hídrica, se localiza esta unidad de paisaje, la más alejada del trazado del acueducto, pero intervisible con él. Se caracteriza por la presencia de los denominados «sotos», espacios llanos o ligeramente cóncavos en el piedemonte serrano, que fueron soporte tradicionalmente a un sistema silvopastoril de cierta importancia y singularidad, muy extendido en los fondos del valle del Lozoya y en la vertiente madrileña del Guadarrama (depresión de Santillana-cerceda, otras zonas), pero menos frecuente en la vertiente segoviana de la Sierra.

El denominado «Soto de Revenga» constituye así un excelente ejemplo del paisaje de campos cercados, con presencia dominante de fresnos en el estrato arbóreo (Fraxinus angustifolia) y ricos pastos, en los que se observan diversas gramíneas y una amplia variedad de vivaces primaverales, como Primula officinalis, la ficaria (Ranunculus ficaria), la violeta (Viola odorata), el narciso (Narcissus bulbocodium), el lamio púrpura (Lamium purpureum), los nazarenos (Muscari comosum y Muscari botryoides), el jacinto silvestre (Endymiom nonscriptus) o las peonías (Paeonia broteroi).

Por la orientación ganadera de estas dehesas, la fracción de cabida cubierta por el fresno y otras especies arbóreas es baja, detectándose una tendencia a la distribución de los resalvos junto a las lindes de separación de las parcelas. Una dinámica observada habitualmente es la regeneración vegetal espontánea en las fincas en desuso o que han reducido apreciablemente su carga ganadera.

Otro elemento característico de este singular paisaje consiste en la propia estructura de la propiedad, con predominio de fundos de tamaño relativamente reducido, que aparecen cercados por paredes de piedra en seco, elementos de gran valor etnográfico y paisajístico, que atribuyen a la unidad su carácter de «defesa» o campo cerrado.

\section{Pastaderos de la Cañada Real Soriana Occidental}

La Cañada Real Soriana Occidental es la vía pecuaria de mayor longitud de toda la península Ibérica y ha constituido durante casi ocho siglos una pieza esencial de la ganadería trashumante. En su largo recorrido, desde Soria a Extremadura, atraviesa parte del término municipal de Segovia, coincidiendo su trazado con el del acueducto durante varios kilómetros.

Dentro del ámbito de estudio, la Cañada Real y sus terrenos aledaños conforman un paisaje homogéneo, caracterizado por la ausencia de cubierta vegetal arbolada y el dominio de los pastizales en una amplia franja de terreno en torno al eje de la vía ganadera y el canal. Este hecho, indisociable del uso ganadero secular que ha soportado la cañada, impidiendo el rebrote y la regeneración del monte, constituye una realidad visual que contrasta con las áreas forestales próximas, cubiertas de pinares o, en su defecto, tapizadas por un bosque mediterráneo de variable densidad según los casos.

En la actualidad, si bien el uso ganadero de la vía pecuaria resulta testimonial, ésta conserva su importancia cultural y ambiental, habiendo adquirido, además, una notable significación como ruta verde y lugar de esparcimiento y uso público, preferentemente en las proximidades del cordel de Santillana. 


\section{Montes de Revenga y Los Hoyos}

Este paisaje discontinuo se localiza al oeste de la localidad Revenga y de la carretera N-603, siendo su límite principal los pastaderos asociados a la Cañada Real Soriana Occidental, aunque se proyecta también hasta la unidad de rampa y el lugar conocido como «Los Hoyos».

El principal descriptor paisajístico de estos terrenos lo constituyen las formaciones de encina, con presencia de ejemplares de porte modesto (salvo contadas excepciones), muy ramificados y con numerosos estolones. El monte no cuenta con un sotobosque significativo, si bien merece un alto valor por su singularidad en el contexto geográfico que ocupa y por la diversidad paisajística que introduce respecto a los terrenos desarbolados contiguos, vinculados a la Cañada Real Soriana Occidental.

Junto con los Pinares de la Acebeda, este paisaje es el que presenta mayor importancia botánica de todos los descritas, quedando englobado en el espacio que integran el LIC y la ZEPA de la Sierra del Guadarrama.

\section{Cerros de Cabeza Grande, El Calvario, La Aldehuela}

Estas modestas elevaciones localizadas en las inmediaciones del núcleo urbano de Revenga, al norte del embalse de Puente Alta, conforman un conjunto de hitos de gran notoriedad física y visual. Por su condición de relieves convexos, de atalayas, adquieren personalidad propia como elementos de contraste paisajístico respecto a la Cañada Real Soriana Occidental, que se sitúa a cota más baja. Por su borde oriental discurre soterrada, aunque reconocible por los registros, la cacera del acueducto.

Estos cerros de naturaleza gneísica constituyen en realidad pequeños horts, levantados e individualizados por sistemas de falla, presentando una característica morfología de domo, con afloramientos rocosos en sus zonas culminantes, que se sitúan siempre por encima de 1.200 m. y llegan a superar en Cabeza Grande los 1.400 m.

Se trata de accidentes topográficos individualizados de los relieves del Guadarrama, de reducidas proporciones relativas, pero relevantes a la escala del ámbito considerado, que marcan la transición entre la extensa y rotunda vertiente segoviana de la Sierra y la rampa, que se proyecta hacia la ciudad de Segovia.

Otra peculiaridad destacable de estos cerros es la densa cubierta forestal que los cubre, mayoritariamente compuesta por encinas de pequeño porte, que se desarrollan sobre los suelos mejores, donde la roca madre no aflora en superficie. La protección histórica de estos montes frente el ramoneo del ganado explica que cuenten hoy con una abundante biomasa, situación que contrasta con la ausencia de vegetación arbolada y leñosa del entorno de la vía pecuaria, pastadero habitual durante cientos de años.

\section{Encinares aclarados de la rampa segoviana}

Este paisaje resulta singular en el término municipal de Segovia y puede considerarse un excelente ejemplo de encinar de la rampa serrana, marcando con claridad la transición entre los montes de encina situados más al sur (cerro de Matabueyes, Cabeza Gatos o Carrera 
Blanca), ya en el término vecino de San Ildefonso, y las dehesas de puro pasto próximas a Segovia.

De los primeros se distinguen por asentarse sobre un relieve con apariencia de cuesta, suavemente basculado hacia el Noroeste, así como por contar con menor densidad de arbolado. De las dehesas de puro pasto las diferencia su acusado carácter forestal, con representación en todos los estratos vegetales (herbáceo, arbustivo, arborescente y arbóreo) y su topografía, de mayores pendientes, aunque litológicamente los dos paisajes adehesados se encuentran sobre al mismo dominio geológico.

La rampa presenta actualmente una clara dinámica de recolonización vegetal, con presencia de abundante matorral serial así como de numerosos estolones en los pies de las encinas, fruto de la reducción de la presión ganadera y del cese del ramoneo, lo que paulatinamente está incrementando la apariencia de «naturalidad» del terreno. Es de destacar la importancia que la misma presenta como hábitat de distintas especies de aves, estando incluida en la ZEPA de la Sierra de Guadarrama.

Este paisaje es accesible desde la carretera que comunica La Granja con Riofrío, a través del cordel de Santillana, y por la propia Cañada Real Soriana Occidental, desde el suroeste, opción que permite obtener las mejores vistas de conjunto.

\section{Dehesas de puro pasto de Aldeanueva y Pradogrande}

Al sur de la carretera que comunica La Granja de San Ildefonso y el palacio de Riaza y a ambos lados del cordel de Santillana, vía pecuaria que discurre algunos cientos de metros en paralelo al trazado del acueducto, se localizan extensas dehesas de puro pasto, comúnmente denominadas «prados» en la terminología local, que se caracterizan topográficamente por su apariencia de dilatada planicie.

La base topográfica y geomorfológica de estas dehesas es también una rampa gneísica con vergencia hacia la ciudad de Segovia y el nivel de base del río Eresma, con suaves abombamientos y vaguadas o navas, escasamente incididas por arroyos como el de la Huelga, la Fuentecilla o Roduelos.

Otra de las características más definitorias de este paisaje es el predominio de grandes fincas con cercas perimetrales y la ausencia casi total de cubierta arbórea, con predominio del estrato herbáceo, situación que responde a antiguos y continuos procesos de roturación para el apacentamiento de ganaderías locales. Este es el caso, precisamente, de la Dehesa de Aldeanueva, de valioso caserío, propiedad que fue adquirida por la Corona en 1768 para acoger una cabaña ganadera destinada al suministro de carne del Real Sitio de San Ildefonso, hasta que fue desamortizada en el siglo XIX.

\section{Eriales entre Nueva Segovia y la variante Sur}

Este paisaje de formas suavemente alomadas está constituido por terrenos de apariencia rural, aunque sin apenas actividad agropecuaria, contiguos a los recientes desarrollos urbanos de Nueva Segovia y nítidamente delimitados al sur por la circunvalación de la capital. Aunque la unidad cuenta hoy con escaso valor ambiental y productivo, hasta hace relativamente poco tiempo formaba parte de las dehesas de puro pasto de la rampa segoviana a las 
que se acaba de hacer referencia y con las que ya se ha roto la continuidad física, funcional y visual, sobre todo por la disposición de la citada variante, que discurre a mayor cota sobre un talud artificial poco permeable.

Por estas razones, el interés de este paisaje radica actualmente en su carácter estratégico a las puertas de la ciudad consolidada, por una parte como espacio con expectativas de urbanización, pero también con un uso público significativo y acceso peatonal a los lugares conocidos como el Lomo y Prado Corral (al norte de la variante, mediante pasos subterráneos) y, por otra, como área de penetración del acueducto a la ciudad.

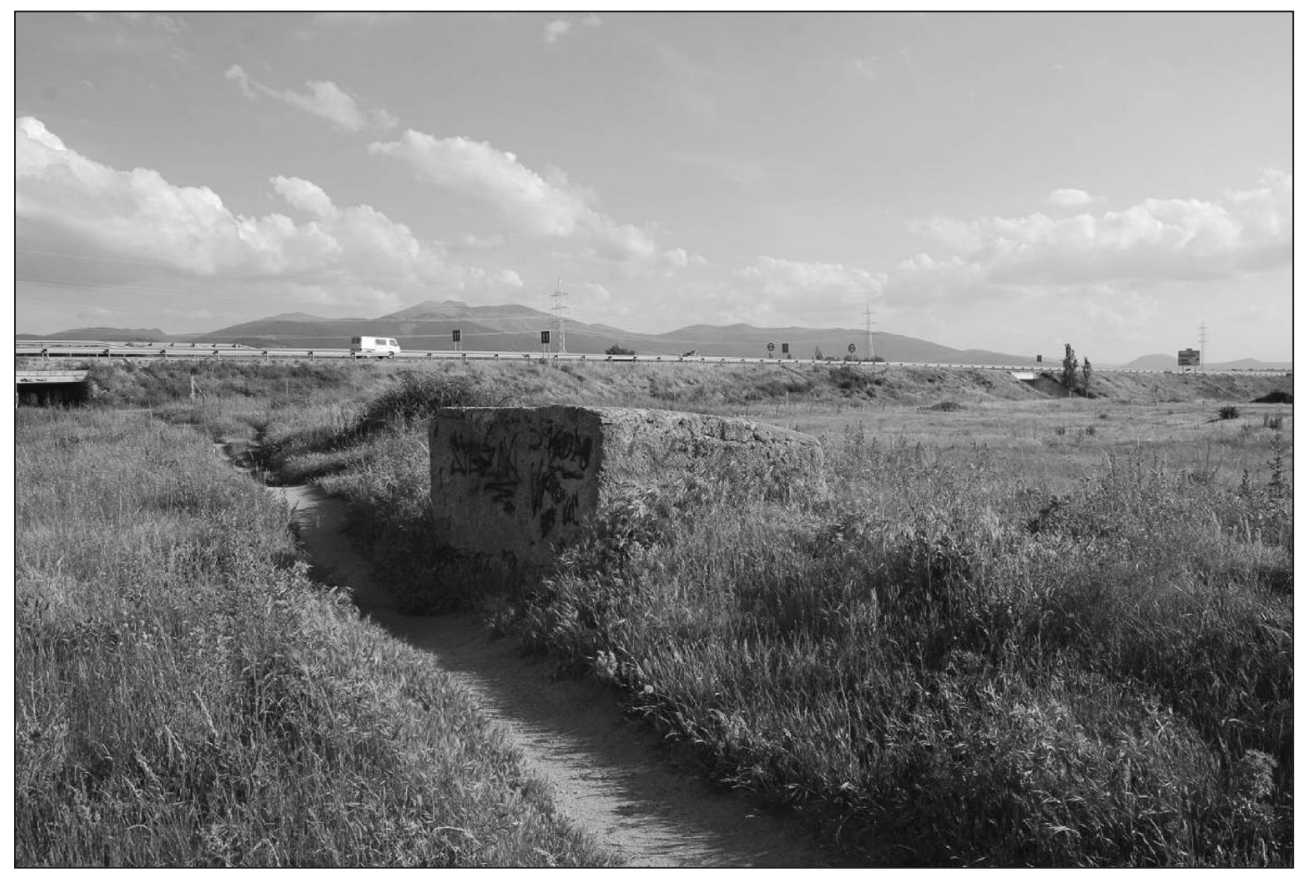

Eriales entre Nueva Segovia y la variante Sur.

\section{LA CONSIDERACIÓN URBANÍSTICA Y TERRITORIAL DE LA TRAZA DEL ACUEDUCTO DE SEGOVIA}

El trazado del acueducto discurre mayoritariamente por el municipio de Segovia, con la excepción de un pequeño tramo, próximo al azud, que lo hace por el término vecino de San Ildefonso. Desde el punto de vista urbanístico, Segovia se rige por su Plan General de Ordenación Urbana (en adelante PGOU), aprobado en 2008. En la escala subregional, el municipio de Segovia está afectado también por las determinaciones de alcance territorial recogidas en las Directrices de Ordenación del Territorio de Segovia y su Entorno (DOTSE), instrumento aprobado por la Junta de Castilla y León en 2005.

El PGOU establece un modelo territorial con importante expansión potencial de la ciudad hacia el sur, afectando a los paisajes de dehesas y eriales y, consiguientemente, al propio 
acueducto, que ni siquiera se menciona en la Memoria del Plan como elemento patrimonial de valor. Esta alternativa de crecimiento urbano se fundamenta, entre otras razones, en la pretendida nueva centralidad que el Plan promueve en torno a la estación del AVE, situada cerca de Hontoria, y en la opción de salvaguardar la fachada monumental de la ciudad desde el norte.

En el caso de los suelos urbanos consolidados de la ciudad de Segovia, la relevancia del acueducto (especialmente a lo largo de su tramo aéreo), el reconocimiento social e institucional con el que cuenta, y las distintas cautelas de protección, garantizan el futuro del monumento. No obstante, el estudio paisajístico llevado a cabo dentro del casco segoviano ${ }^{5}$ pone de manifiesto la existencia de numerosos problemas en relación con las condiciones visuales y los impactos que afectan al monumento. La detección y diagnóstico de tales problemas han permitido establecer criterios y recomendaciones para mejorar la calidad de la escena y el paisaje de la ciudad en su relación visual con acueducto.

Los trabajos de campo se circunscribieron tanto al monumento como a distintas calles y plazas próximas e intervisibles con él, es decir, a evaluar la calidad paisajística del espacio libre público y su arquitectura perimetral (ZOIDO, 2012: 55), definido y connotado por la relación ciudad-monumento. Por la propia naturaleza del acueducto - no se trata de una construcción con espacio interior habitable o visitable - , primó en todo momento la visión «de fuera a dentro», es decir, el análisis de algunas escenas que comúnmente pueden ser contempladas en el casco urbano con el acueducto como elemento destacado. En concreto, se seleccionaron y analizaron un total de 16 vistas representativas, de acuerdo con el procedimiento propuesto por The Landscape Institute del Reino Unido (2002) y por English Heritage (2008). Muy sintéticamente, los problemas más significativos y reiterados son de dos tipos:

- La contaminación de la imagen del acueducto por emplazamientos inadeacuados de señalización vertical y horizontal, mobiliario urbano o elementos vegetales, sobre todo en el tramo en el que la construcción presenta una menor alzada. El estacionamiento de vehículos y los contenedores para recogida selectiva de residuos suponen también, con frecuencia, una interferencia visual significativa.

- La banalización o deterioro del entorno por el estado de ciertas fachadas urbanas que definen la arquitectura perimetral del espacio público y el monumento, en unos casos por su deficiente conservación y en otros, por la existencia de elementos físicos disonantes.

El PGOU previó con buen criterio la redacción de un Plan Especial de las Áreas Históricas de Segovia (PEAHIS), que, entre otros cometidos, debía garantizar y mejorar la integración del acueducto como pieza destacada en su contexto urbano. Dicho Plan ya ha sido redactado y cuenta con Aprobación Inicial desde el 13 de abril de 2010. En él se recomienda además «la recuperación del Acueducto en su tramo no monumental hasta la Sierra (art. 211) y la redacción de un Plan Director del Acueducto (art. 213), que aborde su problemática de manera integral». Aquí nos interesa particularmente la ordenación del denominado «tramo no monumental».

\footnotetext{
5 Trabajo realizado por la paisajista Cristina del Pozo.
} 
En su discurrir kilométrico extraurbano, el acueducto atraviesa, como se ha visto, terrenos muy diversos en su configuración, funcionalidad y valores: áreas forestales; tierras de aprovechamiento agropecuario o eriales periurbanos. Así mismo, la traza del canal, que en algún tramo resulta coincidente con la red de vías pecuarias, se ve afectada por determinadas infraestructuras, como carreteras y circunvalaciones, vías y área de afección del AVE, ETAP, etc.

Estas circunstancias explican que, a efectos de su consideración urbanística, el PGOU haya calificado los suelos sobre los que discurre el acueducto de distintos modos, diferenciando tres grandes categorías, de acuerdo con lo que establece la legislación castellanoleonesa al respecto: Suelos Urbanos, Suelos Urbanizables y Suelos Rústicos. Fuera del Suelo Urbano, el Plan clasifica la mayoría de los terrenos atravesados por la cacera del acueducto como Suelo Rústico, distinguiendo diferentes subcategorías. En cualquier caso, lo importante es que el PGOU no previó un tratamiento especial del acueducto rural y de su entorno, quedando éste protegido sólo «indirectamente» por subcategorías que no consideran específicamente su significado como elemento patrimonial.

Parece razonable que el canal y entorno hubieran sido clasificados como Suelo Rústico de Protección Cultural, de acuerdo con el artículo 36 del Reglamento de Urbanismo de Castilla y León, que establece lo que sigue, pero no fue así:

«Dentro del suelo rústico, deben incluirse en la categoría de suelo rústico con protección cultural:

a) Los terrenos ocupados por Bienes de Interés Cultural declarados o en proceso de declaración, bienes arqueológicos y otros elementos catalogados por los instrumentos de ordenación del territorio y planeamiento urbanístico, así como sus entornos de protección.

b) Los demás terrenos sometidos a algún régimen de protección especial conforme a la legislación de patrimonio cultural, así como sus entornos de protección, en su caso.

c) Los demás terrenos que se estime necesario proteger:

$1^{\circ}$. Por su contigüidad, cercanía o vinculación con los citados en las letras anteriores.

$2^{\circ}$. Por cualesquiera otros valores culturales acreditados, presentes o pasados».

Especialmente preocupante resulta también la situación del acueducto en el periurbano de la ciudad, al sur del polígono Nueva Segovia, donde la traza colinda con Suelos Urbanizables Sectorizados (cuya ordenación de detalle se remite a Plan Parcial), no pudiendo precisarse hasta el desarrollo del citado instrumento cual será el tratamiento que recibirá el histórico canal. En relación con este mismo ámbito se ha observado que algunos viales de conexión (barrio de la Estación-urbanización Peñas del Erizo) o de borde (viario previsto en futuros desarrollos de Prado Corral), propuestos también por el PGOU, afectarían transversalmente al canal, sumándose al efecto barrera que ya ejercen algunas infraestructuras, como la circunvalación o el AVE.

Tampoco las DOTSE han previsto tipo alguno de tratamiento específico para el acueducto, por lo que no es posible encontrar en este instrumento y en esta escala de ordenación las bases en las que fundamentar posibles estrategias de protección y puesta en valor de la 
histórica canalización y de su entorno territorial y paisajístico. Se necesita, pues, una propuesta de conservación integral, como se expone en el último epígrafe del texto.

\section{USO PÚBLICO Y POTENCIAL INTERPRETATIVO DEL ACUEDUCTO Y SUS PAISAJES}

Las distintas características de los terrenos y paisajes por los que discurre el acueducto de Segovia -espacio urbano, área periurbana o zonas rurales y naturales-, guardan una relación estrecha con las diversas formas de uso público del monumento y sus alrededores. Del mismo modo que el recorrido aéreo y monumental es capaz de generar una oferta patrimonial y turística muy importante para la ciudad de Segovia y para la propia región, con decenas de miles de visitas anuales, la traza rural apenas resulta conocida y es poco utilizada, sin que hayan resultando suficientes hasta ahora algunas acciones institucionales emprendidas para llamar la atención sobre su existencia e importancia.

Los estudios realizados sobre este particular permiten concluir que el uso público que soporta buena parte del trazado del acueducto, desde su azud hasta Nueva Segovia, está fragmentado, es intermitente y carece de iniciativas de ordenación e interpretación patrimonial, lo que limita sus amplias posibilidades como conector e itinerario natural y cultural entre la Sierra y la ciudad de Segovia.

En relación con la intensidad del uso público, se ha constatado una gran diversidad de situaciones, con la frecuentación más elevada entre la SG-20 y el trazado del AVE, y unos valores importantes, aunque algo más bajos, entre la vía férrea y la carretera SG.F-7133. En ambos casos, el uso público, peatonal y ciclista, se canaliza a través de la red de caminos y vías pecuarias, coincidentes o contiguos en largos tramos con la canalización del acueducto. Una frecuentación mucho más baja se ha podido comprobar a lo largo de la Cañada Real Soriana Occidental y unos valores modestos también en los pinares de la Acebeda.

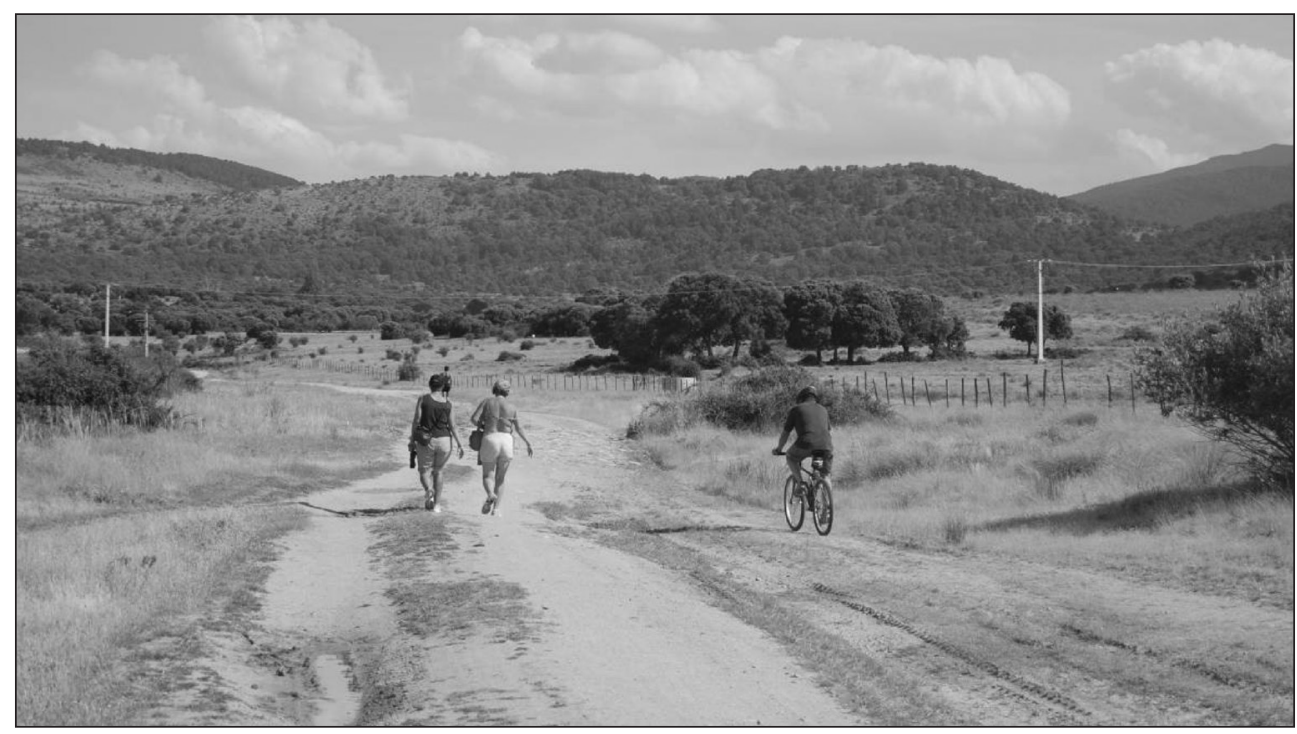

Uso público en el Cordel de Santillana. 
Un hecho muy revelador es que, según las entrevistas a los usuarios que frecuentan estos espacios, el uso público es ajeno, en la mayoría de los casos, a la realidad del acueducto, no contando las personas consultadas con información previa sobre el recorrido del mismo, con la excepción de los excursionistas que realizan el camino hasta su azud, con origen la mayoría de las veces en el embalse de Puente Alto. Las entrevistas han puesto también de manifiesto las siguientes limitaciones del uso público del acueducto y su entorno:

- El mal estado de conservación del azud y de los elementos visibles (no soterrados) vinculados con la obra de ingeniería, tales como caceras, drenajes trasversales o pozos de aireación.

- La ausencia de un programa de señalización adecuado, que permita seguir la traza desde la sierra hasta el llano.

- La falta de actuaciones para la puesta en valor del acueducto y sus paisajes (paneles explicativos, zonas de descanso, miradores, etc.).

\section{UNA PROPUESTA ABIERTA PARA LA CONSERVACIÓN INTEGRAL DEL ACUEDUCTO DE SEGOVIA Y EL DISFRUTE DE SUS PAISAJES}

El estudio presentado en este texto ha permitido abordar, dentro de las iniciativas impulsadas por la WMF ante los peligros que amenazan al Acueducto de Segovia, el estado del bien patrimonial en su unidad y desde una perspectiva territorial y paisajística hasta ahora obviada. Se pretende con ello reducir la ignorancia que pesa sobre la traza rural y complementar las fundamentales aproximaciones arqueológica y de ingeniería histórica para la salvaguarda del monumento.

En el curso de los trabajos se han identificado los problemas más graves que aquejan al acueducto como elemento patrimonial territorial. Pero al mismo tiempo se ha constatado el alto potencial que su recorrido extraurbano ofrece para fortalecer la interpretación del acueducto como histórico viaje del agua, y, a otro nivel, como vinculo entre la Sierra y Segovia, entre la ciudad y los recursos de todo tipo que el Guadarrama le ofrece hoy y le ha aportado durante dos milenios.

Para finalizar, considerando la propia diversidad de los paisajes que el acueducto recorre y la distinta problemática de los terrenos que atraviesa, la propuesta que a continuación se sintetiza responde necesariamente a distintas escalas y enfoques, aunque primando siempre, como se señaló al inicio, la relación entre el monumento histórico y su entorno en clave de paisaje.

Dentro del casco urbano (ámbito muy sintéticamente abordado en esta ocasión), donde el acueducto adquiere su carácter monumental y donde las amenazas directas sobre su entorno resultan objetivamente menores, la propuesta enfatiza en las cuestiones relativas a la mejora y protección de las vistas, concluyendo que cualquier acción por parte del Plan Especial o de los muy necesarios Planes de Gestión demandados por la UNESCO (Troitiño Vinuesa et al., 2011), debería perseguir de manera prioritaria, aunque no exclusiva, la protección escénica del acueducto mediante la gestión activa de las cuencas visuales, lo que implicaría necesariamente la intervención en el espacio público y la correspondiente regulación de usos.

En el perirubano meridional de Segovia, donde los problemas son más acuciantes y el futuro más incierto por falta de previsiones explícitas del PGOU, las propuestas se orientan preferentemente hacia un diseño de los futuros desarrollos urbanísticos capaces de integrar 
correctamente la traza del acueducto como elemento de valor patrimonial. Su preservación y realce contribuirán al mismo tiempo a dignificar y cualificar las nuevas áreas urbanizadas. Hay soluciones muy interesantes desde la jardinería y la arquitectura del paisaje, siempre que el acueducto se libere de la carga de ignorancia y abandono que lo ha maltratado hasta ahora y pase a vertebrar el diseño del sistema de espacios libres de una auténtica nueva Segovia.

Más allá de los suelos urbanizables, en el periurbano rústico contiguo, el reto está no sólo en reducir las afecciones negativas de la traza y garantizar su protección, sino en mantener y mejorar su permeabilidad, reduciendo al máximo el efecto barrera producido por la multiplicación de infraestructuras trasversales. En ese sentido, cualquier crecimiento de la ciudad de Segovia hacia al sur debería considerar y fortalecer el rol de conector territorial entre la sierra y la ciudad que el acueducto ha desempeñado durante siglos, lo que implica que cualquier futuro plan de incidencia territorial tendría que incluir siempre una reserva de suelo suficientemente amplia para preservar esta importante función patrimonial y ambiental.

Por su parte, en las áreas de mayor naturalidad y ruralidad, especialmente en el tramo comprendido entre la carretera que comunica La Granja de San Ildefonso con Riofrío y el azud, donde la conducción atraviesa los paisajes más íntegros y valiosos, la propuesta plantea otro tipo de acciones, preferentemente orientadas a la sensibilización, mejora del uso público y restauraciónconservación de los elementos visibles del acueducto. En ese sentido, el estudio llevado a cabo aporta una amplia batería de acciones dirigidas a mejorar la señalización del trazado y favorecer la interpretación de los «Paisajes del Acueducto», mediante el establecimiento y habilitación de miradores, paneles interpretativos y zonas de descanso, entre otras actuaciones.

Por último, una medida necesaria y de profundo calado para la preservación integral del acueducto sería la extensión de la actual consideración de Bien de Interés Cultural (BIC) a la traza no protegida. De concretarse tal declaración, que fue lanzada ya en la «Jornadas sobre la conservación del Acueducto de Segovia», organizadas en noviembre de 2009 por WMF, en colaboración con el Centro de Patrimonio Mundial de la UNESCO, resultaría muy conveniente la definición de un entorno de BIC generoso, de acuerdo con el método y los valores paisajísticos tratados en este estudio y que, como tal, debería ser incorporado al instrumento de planeamiento general en vigor.

\section{BIBLIOGRAFÍA CITADA}

CABALLERO SÁNCHEZ, J.V. y ZOIDO NARANJO, F. (2008): «Formación y desarrollo de una línea de investigación: la dimensión paisajística de los conjuntos arqueológicos». Cuadernos Geográficos de la Universidad de Granada, n 43(2008-2), pp. 181-198.

CENTRO DE ESTUDIOS PAISAJE Y TERRITORIO (2011): El paisaje en el Conjunto Arqueológico Dólmenes de Antequera. Sevilla, Junta de Andalucía, Instituto Andaluz de Patrimonio Histórico, 215 pp.

CHOAY, F. (2007, $1^{\text {a }}$ ed. 1992): Alegoría del patrimonio. Barcelona, Editorial Gustavo Gili, $263 \mathrm{pp}$.

CHOAY, F. (1993): «Alegoría del patrimonio. Monumento y monumento histórico». Arquitectura Viva, no 33, pp. 15-21.

ENGLISH HERITAGE (2008 y 2011): Seeing History in the View: A Method for Assessing Heritage Significance within Views. London, English Heritage, 61 pp. 
http://www.english-heritage.org.uk/content/publications/publicationsNew/guidelines-standards/seeing-history-view/seeing-history-in-view.pdf.

FERNÁNDEZ CASADO, C. (1972; 2a ed. 2008): Acueductos romanos en España. Madrid, Colegio de Ingenieros de Caminos, Canales y Puertos, Colección ciencias, humanidades e ingeniería, $283 \mathrm{pp}$.

FERNÁNDEZ TROYANO, L. (2008): «Presentación de la segunda edición», en FERNÁNDEZ CASADO, C.: Acueductos romanos en España. Madrid, Colegio de Ingenieros de Caminos, Canales y Puertos, Colección ciencias, humanidades e ingeniería, p. 9.

GALLARDO RAMÍREZ, A. (1992): Supervivencia de una obra hidráulica. El Acueducto de Segovia. Madrid, Colegio de Ingenieros de Caminos, Canales y Puertos. Colección de Ciencias, Humanidades e Ingeniería. Madrid, 1992, 295 pp.

JURADO JIMÉNEZ, F. (2001): «El acueducto romano de Segovia». OP Ingeniería y Territorio, $\mathrm{n}^{\circ} 57, \mathrm{pp} .14-23$.

http://www.ciccp.es/revistait/portada/img_portada/issue_111/pdf/OP_57.pdf.

MANUEL VALDÉS, C. (2008): «Propiedad, usos y gestión de los montes de Valsaín», en I Jornada de Divulgación de Estudios Científicos Sobre los Montes de Valsaín. Valsaín, CENEAM, $17 \mathrm{pp}$.

http://www.ciccp.es/revistait/portada/img_portada/issue_111/pdf/OP_57.pdf.

MARQUÉS MARTÍN, I. (2010): El Acueducto de Segovia, Patrimonio de la Humanidad. Segovia, Ayuntamiento de Segovia, cuadríptico.

MARTÍNEZ DE PISÓN, E. (1976): Segovia. Evolución de un paisaje urbano. Madrid, Colegio de Ingenieros de Caminos, Canales y Puertos, 436 pp.

MATA OLMO, R. (2010): «La dimensión patrimonial del paisaje. Una mirada desde los espacios rurales», en MADERUELO, J. (Dir.): Paisaje y Patrimonio, CDAN, Huesca, pp. 31-73.

MATA OLMO, R. y GALIANA MARTÍN, L. (2008): «Ordenación y gestión del patrimonio cultural y el paisaje. La experiencia del Plan Especial de la Sierra de los Molinos en Campo de Criptana. Cuadernos Geográficos de la Universidad de Granada, nº 43 (20082), pp. 199-225.

RIEGL, A. (1987; $1^{\text {a }}$ ed. 1903): El culto moderno a los monumentos. Boadilla del Monte, A. Machado Libros. (citado por CHOAY, F.)

THE LANDSCAPE INSTITUTE (2002,2 ${ }^{\mathrm{a}}$ ed.): Guidelines for landscape and Visual Impact Assessment. London, Institute of Environmental Management \& Assessment. Spon Press, $176 \mathrm{pp}$.

TROITIÑO VINUESA, M.A., GARCÍA HERNÁNDEZ, M. y CALLE VAQUERO, M. DE LA (2011): «Las actividades turístico-recreativas en los planes de gestión de los sitios Patrimonio Mundial. El caso de Aranjuez, Paisaje Cultural de la Humanidad». Cuadernos de Turismo, $\mathrm{n}^{\circ} 27, \mathrm{pp} .907-929$.

ZAMORA CANELLADA, A. (1995): El Acueducto de Segovia. Segovia, Academia de la Historia y Arte de San Quince, $91 \mathrm{pp}$.

ZOIDO NARANJO, F. (2012): «Paisaje urbano. Aportaciones para la definición de un marco teórico, conceptual y metodológico», en DELGADO VIÑAS, C., JUARISTI LINACERO, J. y TOMÉ FERNÁNDEZ, S. (Eds.): Ciudades y paisajes urbanos en el siglo XXI. Santander, Ediciones Librería Estudio, pp. 13-91. 